\title{
INFORMATION SUPPORT SYSTEM FOR TECHNOLOGY OF APPLYING THE MULTI-COMPONENT NANO-STRUCTURED PROTECTIVE COATINGS
}

\author{
Eugene Kopytov ${ }^{1}$, Alexander Urbach ${ }^{2}$, Valery Kutev ${ }^{1}$, \\ Vladimir Labendik, Sergey Yunusov ${ }^{1}$ \\ ${ }^{I}$ Transport and Telecommunication Institute \\ Lomonosova str.1, Riga, LV-1019, Latvia \\ Ph.:(+371)67100614.E-mail: kopitov@tsi.lv,lvp@tsi.lv,yunusov@inbox.lv \\ ${ }^{2}$ Institute of Transport Vehicles Technologies of the Riga Technical University \\ Lomonosova str.1, Riga, LV-1019, Latvia \\ E-mail: aleksandrs.urbahs@rtu.lv
}

\begin{abstract}
The given article considers the process of arranging informational support in carrying out experiments on applying the nanostructured protective covering on different materials. For nano-coverings coating, there is used special plant, which is given different modes of performance. For accounting all parameters and for accumulating the data, which characterize the results of the experiments, there is suggested an information system which employs the database designed for these purposes. The authors suggest an approach to defining the main requirements and functions of an information system on the basis of functional simulation and consider the issues of its realization. This information system also allows processing the results of the experiments with the use of statistical methods. The analytical module of the information system is supposed for solving the tasks of determining the thickness of covering depending on the specified mode of plant operation (pressure, voltage, current); it is also employed for determining what operation mode is required for receiving the covering with certain designated properties and parameters. Consequently, the task of scientific prognostication of the covering properties is solved.
\end{abstract}

Keywords: functional model, database, parameters of coverings, mode of performance nanotechnology, information system, analytical processing

\section{Introduction}

The nanosystems material study faces the complex of scientific and technological problems, solution of which is to be directed not only on the research of scale factor (such as decrease of particles, elements and other structures size) but also investigation of landmark phenomenon which is the distinctive feature of nanoscale.

The nano-technologies designed in the latest decades comprise such technology as the process of vacuum plasma deposition of nano-coating. The nano-materials conventionally involve disperse and massive materials with some content of structural elements (grains, crystals, blocks, clusters), if their geometrical sizes do not exceed $100 \mathrm{~nm}$ at least in one dimension and possessing the brand new properties, and functional and operational features.

Nanotechnologies are technologies operating by sizes of nanometer order. It is an extremely small size which is hundred times less than the visible light wave-length and comparable with the size of atoms. Plasma deposition of nano-coating having the minimal depth from 1 nanometer $(0,001$ micron) to 1000 nanometers (1 micron) provides the substantial increase of functional properties of different items. The thickness range of applied coating measured with nanometers consequently corresponds to the values of nanoworld and marks this process as a nanotechnological process. Besides scale factor of applied coating the procedure of nanoplasma deposition has a nanosized time of certain stages of interaction with the worked up material; this time is responsible for the quality of coating, specifically rates of heating and cooling the basic metal. The substrate surface under the coating in the layer till 1000 nanometers ( 1 micron) depth is heated by about $200^{\circ} \mathrm{C}$ for $10^{-2}$ nanosecond, and then is cooled by initial temperature for 1,0 nanosecond [1].

New construction materials are the cornerstones of transport and especially aviation transport success. More than 50 years ago there functioned the scientific school under the supervision of Professor 
V. Prosvirin in the Riga Civil Aviation Engineering Institute (RCAEI). This school operated on the base of a material science laboratory and investigated the protective coating. After disassembly of Riga Aviation University in the end of 90 s the academic staff and employees of the university changed the place of work to other higher education establishments, preserving their scientific potential.

In 2010 the researchers of Riga Technical University (RTU) in cooperation with the researchers of Transport and Telecommunication Institute (TTI) and Rezekne Higher School Establishment (RHS) on the base of laboratory of Department of Technologies and Repair of Transport Technologies Institute (ex-lab of Material Science of RCAEI) stared with the Joint research project of Latvian Council of Science "Development of Technology for Industrial Multicomponent Nanostructure Sheeting" [2]. The results of some project's tasks fulfilment are presented in this paper.

\section{Technologies of Applying and Quality Control of Nanocoatings on the Machines Components Employing the Technological Process of Ion-plasma Coating}

The first stage of investigation is devoted to the general possibility to arrange the contemporary base and system of processing the information on research of one of nanocoating creation directions, specifically wear-resistance coatings. The most efficient areas of employing the plasma deposition of wear-resistance nano-coating are tools hardening and industrial equipment hardening.

Composition and properties of wear-resistance coatings significantly depend on technique and technology of their applying. These methods are divided into two big groups: physical (PVD) and chemical (CVD).

Under the physical deposition (method PVD, or physical vapour deposition) the coating material is transferred into gas-phase from solid phase as a result of evaporation affected by thermal energy or as a result of dispersion affected by kinetic energy of material particles collision. Energy, dispersion and density of particles flow are determined by applying method, by process parameters and by particles source form. Applying the coatings with an employment of PVD method under rather low temperature regime (up to $450^{\circ} \mathrm{C}$ ); this fact practically does not imply any restrictions on the materials, used for coating applying. All the PVD processes take place in vacuum or in atmosphere of operating gas under rather low pressure (about $10^{-2} \mathrm{mbar}$ ). It is necessary for facilitation of particles transfer from the source (storage plate) to item (substrate) with minimal number of collisions with atoms or molecules of gas. The same condition determines the necessity of particles direct low. As a result the coating is applied only to the part of item directed to the source of particles. One of basic factors determining the quality of coating applied by physical vapour deposition method is the purity of initial materials, the required level of vacuum and purity of reaction gas $[3,4]$. All these processes are divided into two big groups: the processes utilizing the evaporation procedure, and the processes utilizing the sputtering procedure.

Among all facilities dealing with the sputtering procedure, the most widely used ones are the plants on the basis of magnetrons (MSIP - Magnetron Sputtering Ion Plating). In the process of implementing the high voltage in the atmosphere of inert gas (it is argon as a rule) the glow-discharge occurs. The inert gas ions from plasma usually possess the high energy and impact the target, inserted as a cathode. At the expense of impact impulse the release of the material takes place, and then it is sputtered escaping the intermediate liquid stage.

The magnetron method is the sub-kind of the cathode sputtering method, which assumes that the layer of plasma is formed at the surface of the sputtering cathode (the target) employing the crossed magnetic and electrical fields. The density of this plasma is significantly bigger compared to the common (non-magnetic) systems of cathode sputtering. Accordingly, there is a sufficient growth of the density of ion flow on cathode and the velocity of cathode sputtering. The magnetron method allows applying the wide spectrum of covering of various metals and their compounds with the high uniformity of properties including the highly rigid durable coatings. The sputtered particles contain the neutral atoms on $75-95 \%$, that is why the bottom layer (a detail, an item, a tool, etc) is heated faintly, and this permits to deposit the coatings on the detail having the low fusion temperature (low-melting-point metals and alloys, plastics, organic substances). The magnetron sputtering is supposed to be the prospective method and at the same time it is a method which has been well-tried and developed in the laboratory of Department of Technologies and Repair of Aeronautics Institute in Riga Technical University [5]. External view of magnetron plant is shown on Figure 1. In its turn Figure 2 demonstrates the scheme of vacuum ionplasma sputtering the coatings on the details surfaces. 


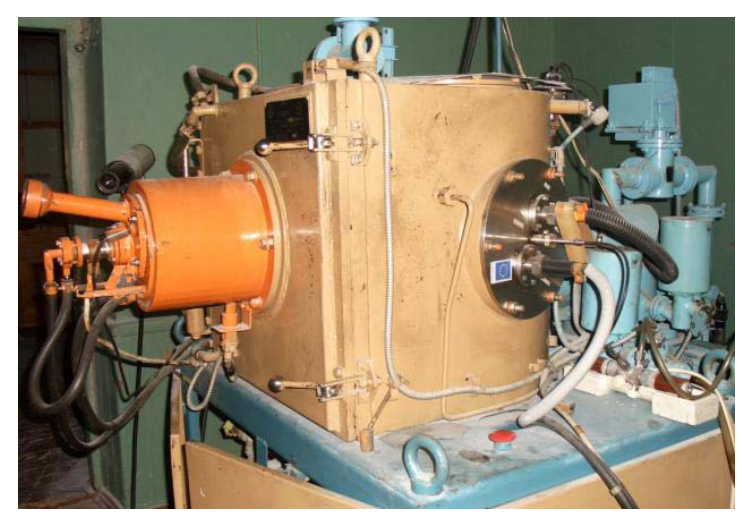

Figure 1. External view of magnetron plant

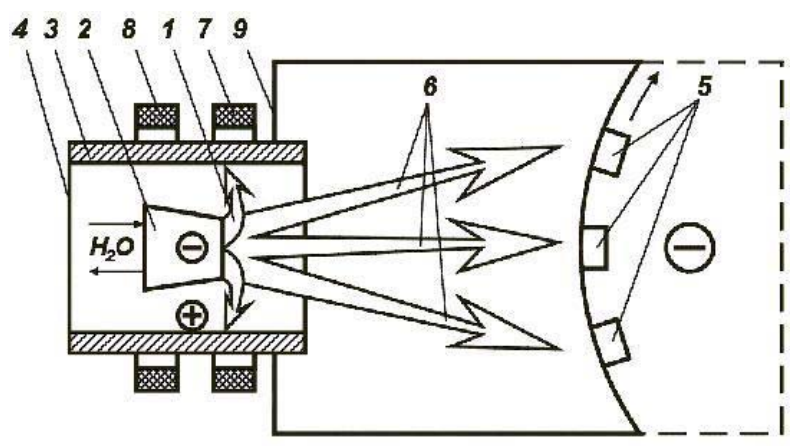

Figure2. Vacuum ion-plasmas sputtering installation scheme:

1 - plasma, 2 - cathode (arc evaporator), 3 - circular anode, 4 - gun, 5 - worked parts, 6 - sputtered material, 7 - focusing magnetic coil, 8 - regulating magnetic coil, 9 - vacuum chamber.

Thickness and evenness and accordingly the quality of applying coating depend on the process control power: position of a detail on the spinning table, choice of sputtering modes (see Figure 3 ).

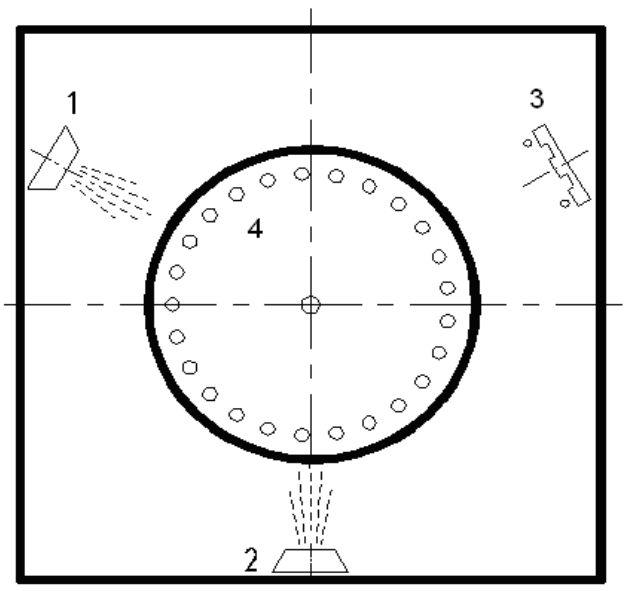

Figure 3. The scheme of evaporators location on the modified vacuum installation: 1, 2 - arc-jet evaporators; 3 - magnetron; 4 - turntable

The technological parameters of the coating applying process make and determine the procedure of coatings quality control; nevertheless, it is necessary to know the way how the precipitation process technology provides the quality of precipitated coating with the specified degree of accuracy. 
The fundamental parameters describing the precipitated coating quality are as follows:

- coating thickness;

- microhardness of coating or composition of coating-backing, GPa;

- strength of coating and substrate adhesion;

- roughness of the surface with coating;

- coefficient of friction.

These parameters are related with the employment purpose of coated details. The mentioned input and output parameters of nano-coating plasma deposition are the principal ones in the process of estimating and optimising this technology. That is why the subsequent research stages assume possibility of organising the enhanced database under the condition of equipping the laboratory of Department of Technologies and Repair of Aeronautics Institute in RTU with other research plants for complex evaluation of obtained coating samples. The electronic microscope and the diffractometer ADP-1 are used in the laboratory for complex evaluating the quality of applying the nano-coatings with specified properties. Nevertheless the output analogue signal of this diffractometer was directed to the computer M-6000 and data plotter. The investigated detail of this diffractometer can be irradiated and rotated on the operating table for $60^{\circ}$ at a pitch of $0,001^{\circ}$. Consequently, under investigation of one sample only (at one sectional view of, for instance, compressor or turbine blade), the massive of 60000 signals, describing the deposition uniformity and coating structure, is obtained. It is necessary to compare the amplitudes of output signals with the base (reference) values for determining the quality of coating with preset properties [6].

At the first stage of present research the tasks of the authors were the following: to develop the means of transforming the analogue signals from diffractometer into the digital ones; to analyse the structure and volume of obtained information and determining the possibilities of results accumulation in the specialised database of personal computer.

\section{Development of Automated Digital Measuring System of Registration and Control of the X-rayed Diffractometer Data}

The proposed system of automated registration and taking off the data is oriented on detection and displaying the signal of basic goniometer (BG) general purpose, the functional blocks of which are the principal components of the X-rayed diffractometer. The goniometer implements the principle of focusing the X-ray radiation, dispersed by investigated sample [7].

Registration of output signals BG-0 is implemented in the analogue system employing the laboratory compensation recorder. The form of presentation is shown on Figure 4.

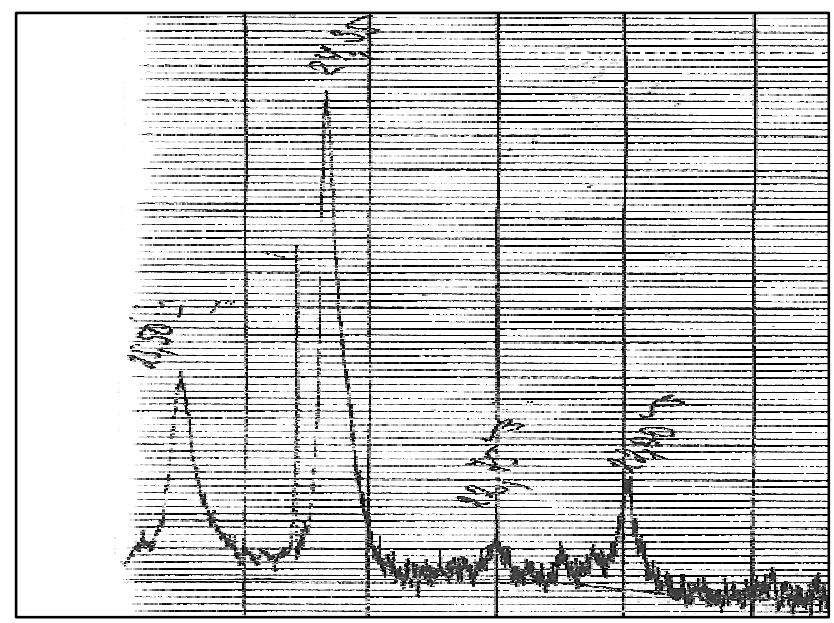

Figure 4. The fragment of amplitude registration of output signal of radiation detector of the X-rayed diffractometer

For processing the measurement results according to the recorder data, done with employment of the X-rayed diffractometer, the combination of at least two operations is necessary:

- fixation of maximum angles of recorder output signals;

- estimation of maximum values of output signals in relative units depending on the equipment settings (on Figure 4 the maximum values are marked with corresponding notes). 
Formerly both operations were executed manually. This fact significantly increases the laboriousness of the measurement results processing procedure and does not provide the acceptable accuracy of measurements. Moreover, the process of preliminary setting the equipment is significantly complicated.

For eliminating the above mentioned defects it is offered to combine the measuring equipment and the system of automated registration and data taking off, the principal functions of which are done with employment of analogue-digital converter (ADC) of output signals of radiation detector and personal computer (PC of Notebook type), presenting and processing the signals at software level.

The structural scheme of the created system of automated data registration and data taking off of the X-rayed diffractometer is demonstrated on Figure 5. It comprises the following blocks: output signal coupler of radiation detector, scaling amplifier, analogue-digital converter (ADC), triggering pulse generator, buffer register, USB interface and PC (notebook).

The graphic block comprises the set of programmes providing the representation of sampling of measuring equipment signals on PC display in the view convenient for tuning the measuring equipment and further processing the measurements results.

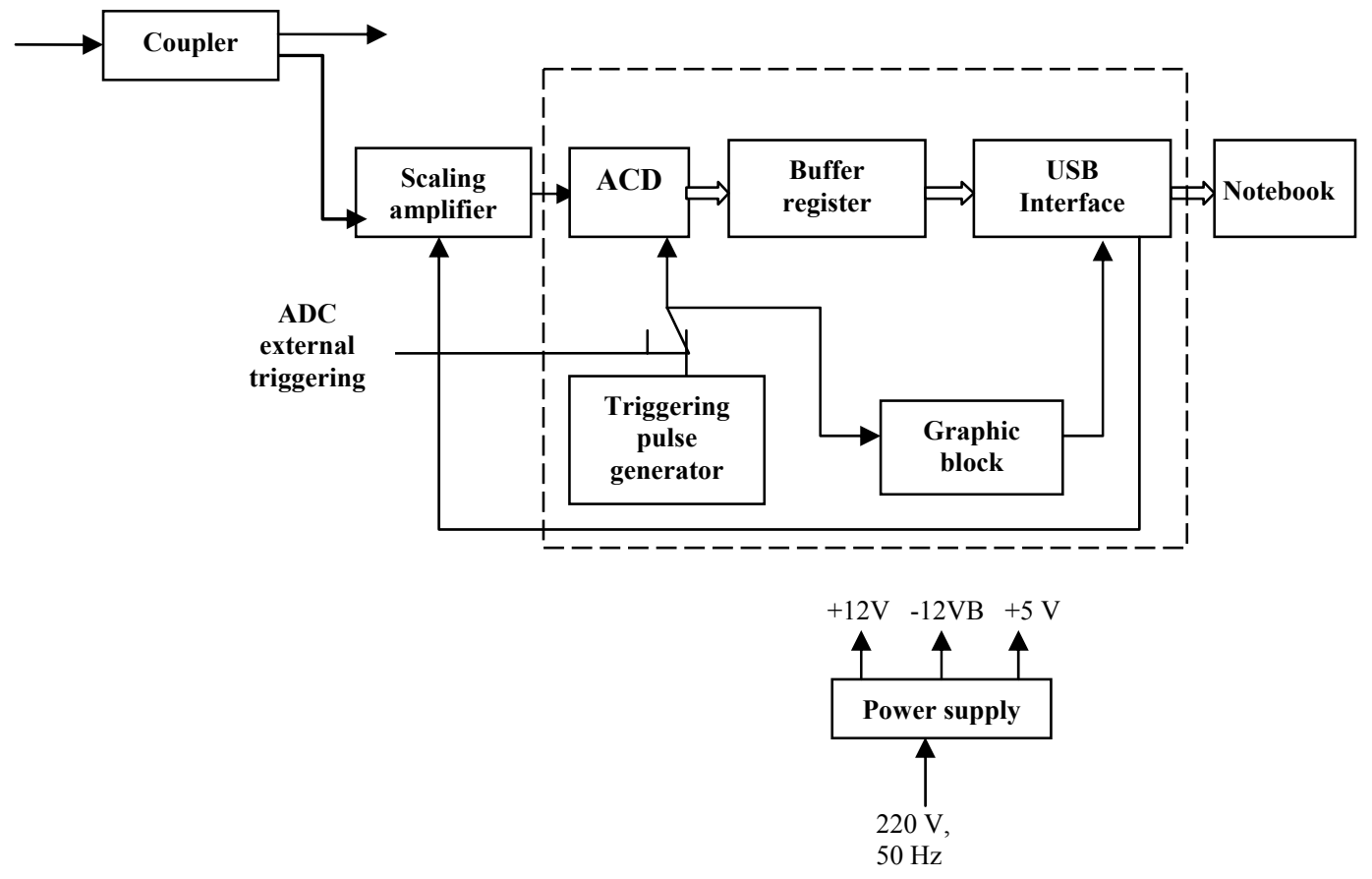

Figure 5. Structure scheme of automated registration system and the X-rayed diffractometer data taking off

The conversion device, marked with dashed line on Figure 5, is implemented on the basis of microcontroller AT90USB1287, comprising integrated ADC, generator and USB interface with power supply of $+5 \mathrm{~V}$. AT90USB1287 is an economical 8-rated CMOS microcontroller implemented on the basis of high-performance architecture AVR RISC. By means of executing the majority of instructions at one synchronisation period the microcontroller AT90USB1287 reaches the efficiency of 1 million operations per second at $1 \mathrm{MHz}$ of timing frequency, allowing optimisation of ratio of required power and promptitude.

Microcontrollers are produced according to the technology of high-density non-volatile memory. The procedure of programming the integrated flesh-memory allows re-programming it inside the system via the consecutive interface SPI with employment of non-volatile memory programmer or with integrated boot programme, executed by AVR core. The boot programme can utilize any interface for downloading the programming code into flash-memory. By means of dividing the flash-memory into booting sector and applied programme sector, the actual possibility of reading in the course of recording when the booting code is still executed and the sector of applied programme is updated.

All the exchanges (transactions) in USB consist of 3 packages. Every transaction is planned and starts due to the initiative of the host-controller, which is comprised in personal computer. The host-controller 
sends the marker package (of token type), describing the type and direction of transaction, address of USB device, number of final point.

There is only possible exchange between the final point of device and host for every transaction. The source of data, determined by marker, transfers the package. After successful receiving the package, the data receiver sends the package of confirmation (package of Handshake type). Since the maximum frequency of ACD discretization is $10 \mathrm{~Hz}$, it is possible to use a low-speed mode of operating the USB bus bar USB LS (Low-speed), providing the transmission capacity of 1,5 Mbps. Moreover, for employing the drivers integrated into the operational system PC, it is reasonable to consider ACD as a HID-device (Human Interface Device), the maximum exchange speed of which is $64 \mathrm{Kbps}$.

The graphics block is a standard window application with WinAPI interface, created in the environment of object oriented programming Borland Delphi 7 employing the compiler of Object Pascal language. The user's interface, located in the right-hand part of the programme principal window, presents several logically separated groups of buttons and windows of settings.

The following algorithms, implemented in the block of programming graphics, are employed for presenting and processing the measuring device signals: the amplitude indication of signals, colour brightness indication of registered signals levels, processing the signals, using the Hilbert transformation [8,9] (see Figure 6).

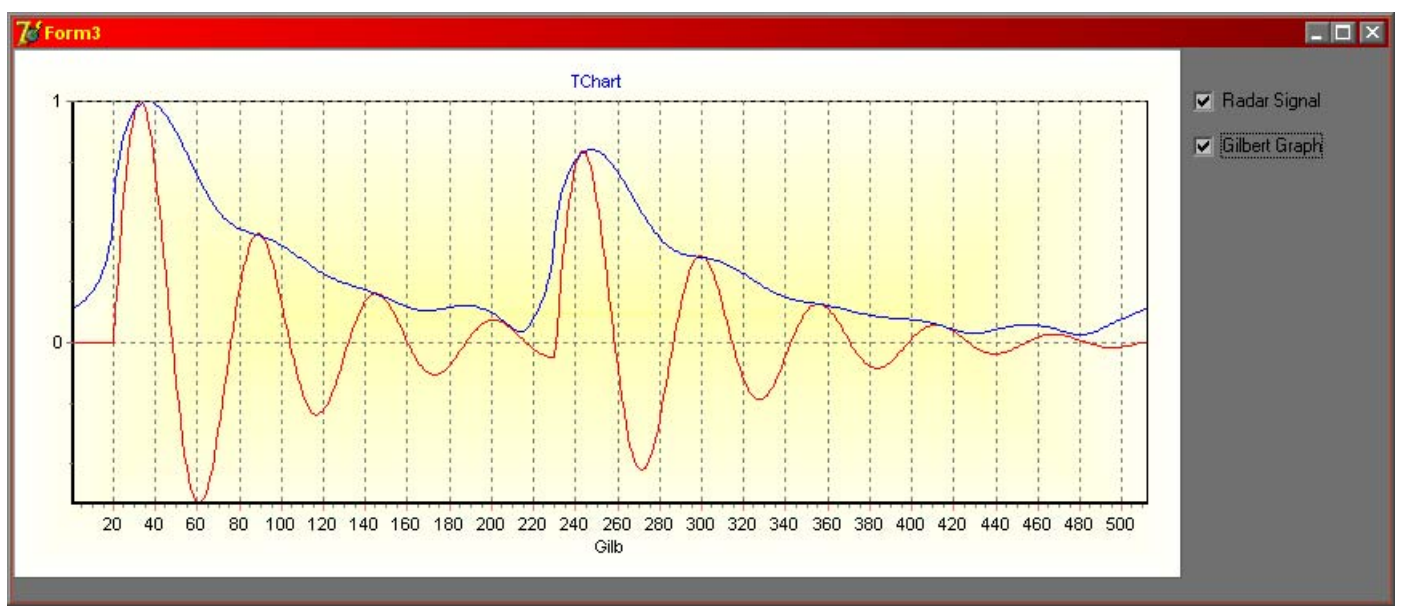

Figure 6. The amplitude registration of signals employing the Hilbert transformation

\section{Development of Information System of Statistical Processing the X-rayed Diffractined Data Analysis of Results of Experiments on Producing Nano-coatings}

The research group of the Institute of Transport Machinery Technologies in Riga Technical University is engaged in the investigation of issues of applying thin layers (nanocoatings) on various items for a long time. Information on the results of carried experiments is kept in different ways, partly in electronic form, partly in hard copies. The diffractometer, the signals of which are recorded by the recorder, are used for measuring then layer thickness and evenness. The diffractogramme decoding is done manually with employment of special tables, and this process demands the experience specialist's labour and substantial time. The automated measuring set is in the process of developing for solving the problem of measuring the depth of layer; this set is supposed for digitisation of the diffractometer analogue signal and registering the measurements values in the database; this allows determination of the layer thickness in automated mode.

The measurement of the following parameters is necessary for evaluating the quality of precipitated coating:

- coating thickness;

- microhardness of coating or composition of coating-backing, GPa;

- strength of coating and backing adhesion;

- roughness of the surface with coating;

- coefficient of friction.

The below listed parameters can be shown for comparative characteristics of the sample parameter values before and after applying the layer: 
- hardness;

- $\quad$ surface roughness;

- friction coefficient.

The experiments are carried out in a special plant designated for coating applying. Two arc evaporators and one magnetron dispenser are used as the sources of evaporation material. Every experiment is carried out for producing the coating for certain purpose, for instance, wear-resistant, heat-resistant, ornamental, smooth, special, and others. Several samples can be obtained in the same experiment. The following data are registered in the database: experiment number, date of carrying, purpose, executor, experiment description.

The samples are registered, they are numbered, then the information on them is put into database: type of sample (detail, plate, etc), then photo before an experiment, type of material (steel, copper, type of alloy). The sample is set in the sector of plant, its position is registered (vertical or horizontal), if there is spinning (yes/no). There are five sectors in the plant: four peripheral and one central. The following data are registered in the database after carrying out the experiment: electric conductance, electrochemical conductance, hardness, colour, roughness and photo with layers structure. The first stage of an experiment consists of heating the plant for providing the normal adhesion for applying coating on the sample.

The process of heating and modes of applying the coating are described with the following parameters: sputtering material, current intensity, draw-down pressure, gases pressure (argon, nitrogen), temperature, reference voltage and time. Thickness, hardness and deposition rate are fixed for every layer after experiment. Registration and processing the diffractometer results in the database are absent; they will be added only after the solution of technical problems connected with equipment development and its combination with diffractometer.

The presented analysis allows formulating the following business requirement towards the developing system: the specialists of the research group need the information system capable of providing the registration and accumulating information on the conducted experiments, processing data, received from the measuring devices and the complex statistical processing of the obtained results. This system allows increasing the quality of measurements processing, to register and store centrally the information on investigated samples, to take the statistical analysis of the obtained results.

The functional model of the coating applying process has been developed for finding out the full set of functions and tasks of the projected information system. The functional model in the framework of formalization allows gathering the information on the coating applying process in so called "information pile". The functional model has been constructed on the basis of the system structural analysis with implementation of the method IDEF0 [10] using the package BPwin4.0 (see Figure 7)

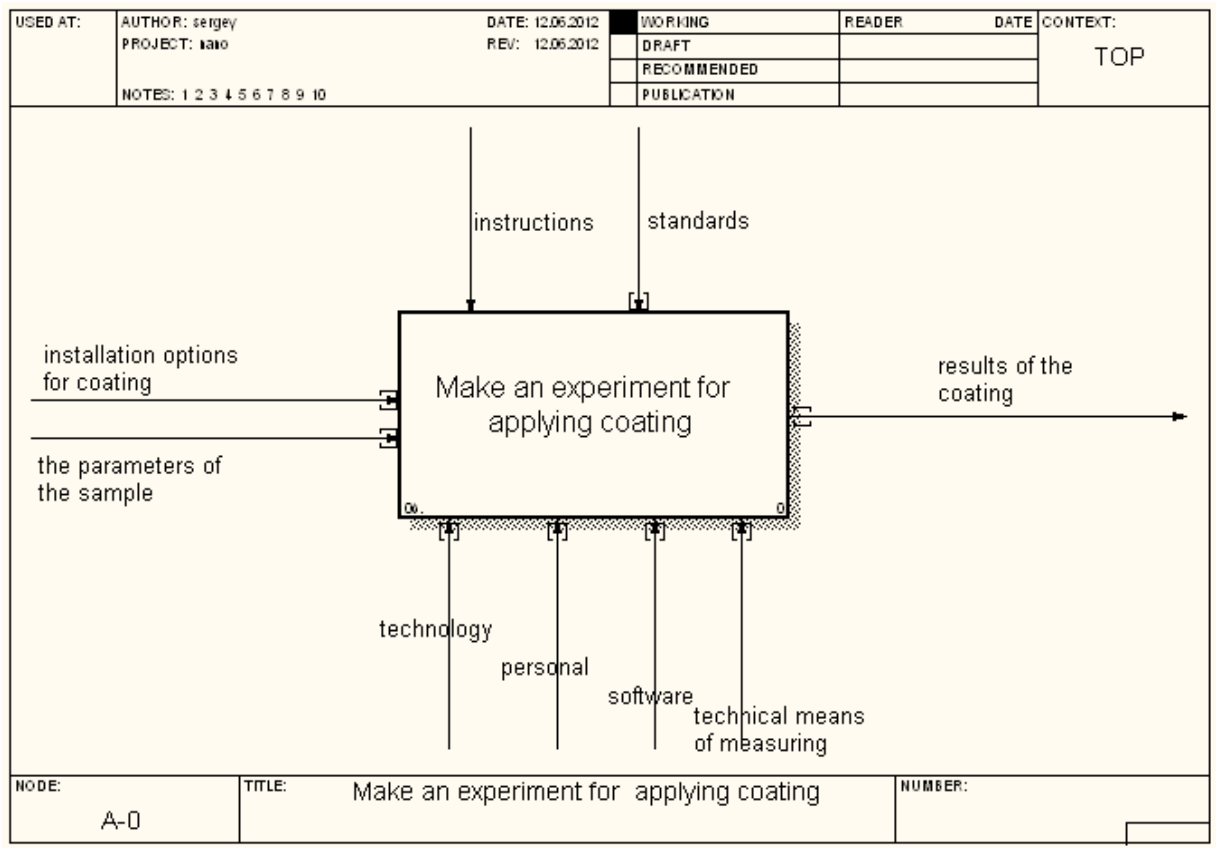

Figure 7. Functional model of coating application process 
The received model has been presented in details in accordance with the tasks solved in the process of applying the coating and contains the following functional blocks (see Figure 8):

- to prepare the sample - the sample is prepared for experiment, the measurements of parameters are found out and entered into the database.

- to apply the nano-coating - the sample is put into the plant, the necessary values of parameters for coating applying are preset and fixed in the database.

- to measure the parameters after the coating application - the measurement of the coating application uniformity, hardness, friction coefficient and strength of its adhesion with its backing is done with implementing the measuring devices.

- to enter the results into the database - this functional block gathers all the data in the experiment process into the "information pile" for consecutive processing and analysing.

- to process the results - the accumulated information is processed with statistical methods employment, then the analysis is done, and moreover, the user has possibility to receive the interesting information from the database in the form of output papers on the basis of the created query.

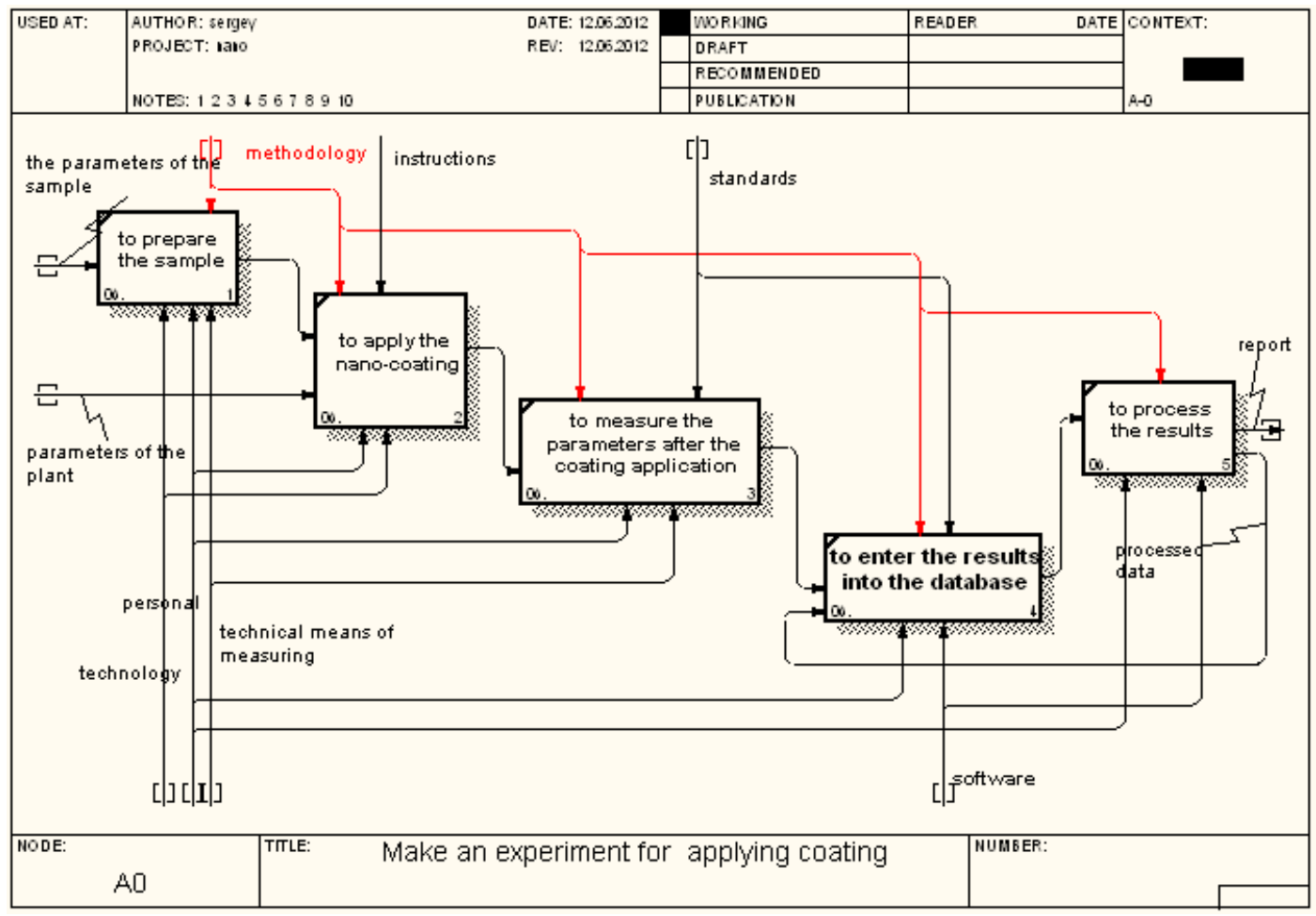

Figure 8. Functional detailed model of coating application process

The process of applying the coating comprises several stages. First, the information about the sample before applying the coating is registered in the database, the sample is numbered, its material or alloy is determined, the photo of this sample is taken, and the initial hardness, roughness and friction coefficient are measured with employment of measuring devices.

Then the sample is placed into the plant for applying the coating, and the parameters of setting mode are fixed (temperature, pressure, magnetic field intensity, length of the process, etc.) and the material applied on the sample.

The evenness and thickness of coating after its applying are examined with an employment of the diffractometer. The parameters, describing the quality of coating applying, can also be measured: hardness, friction coefficient and strength of coating and backing adhesion, the chemical composition of coating. The data of measurements are registered in the database with implementation of ACD.

The database accumulates the information on the process of applying the coating on the sample. The statistical analysis takes place on the basis of accumulated information and in accordance with chosen statistical methods. The system forms the necessary final reports.

The database model has been developed on the basis of functional model; it comprises the entity and their attributes, presented in the Table 1. 
Table 1. Database phenomena and attributes

\begin{tabular}{|c|c|c|}
\hline Entity & Attribute name & Property \\
\hline \multirow{6}{*}{ Sample } & ID_sample & Primary key \\
\hline & Material & \\
\hline & Picture & \\
\hline & Primary key & \\
\hline & Roughness & \\
\hline & Friction coefficient & \\
\hline \multirow{4}{*}{ Coating material } & ID_material & Primary key \\
\hline & Material & \\
\hline & Quantity & \\
\hline & Chemical composition & \\
\hline \multirow{7}{*}{ Sample parameters } & Hardness & \\
\hline & Roughness & \\
\hline & Layer thickness & \\
\hline & Friction coefficient & \\
\hline & ID_sample & Foreign key \\
\hline & Layer chemical composition & \\
\hline & Strength of adhesion & \\
\hline \multirow{7}{*}{ Setting mode } & ID_setting mode & Primary key \\
\hline & Temperature & \\
\hline & Pressure & \\
\hline & Magnetic field intensity & \\
\hline & Time & \\
\hline & Angle of rotation & \\
\hline & Pitch of rotation angle & \\
\hline \multirow{5}{*}{ Coating applying } & ID_coating applying & Foreign key \\
\hline & ID_setting mode & Foreign key \\
\hline & ID_material & Foreign key \\
\hline & ID_sample & Foreign key \\
\hline & Date of application & \\
\hline \multirow{3}{*}{ Measurements of diffractometer } & ID_sample & Primary key \\
\hline & ID_coating applying & \\
\hline & Amplitude of a signal & \\
\hline \multirow{5}{*}{ Test } & ID_tests & Primary key \\
\hline & Date of test & \\
\hline & Type of test & \\
\hline & Test description & \\
\hline & ID_sample & Foreign key \\
\hline \multirow{6}{*}{ Test results } & Hardness & \\
\hline & Layer thickness & \\
\hline & Friction coefficient & \\
\hline & Roughness & \\
\hline & Strength of adhesion & \\
\hline & ID_Test & Foreign key \\
\hline
\end{tabular}

Database Implementation. The database has been implemented on the basis of functional model using MS Access 2007 [11]. This database management system (DBSM) quite corresponds to the solved tasks on saving, storage and processing the information under the condition of conducting experiments. The created data model is presented below on Figure 9. 


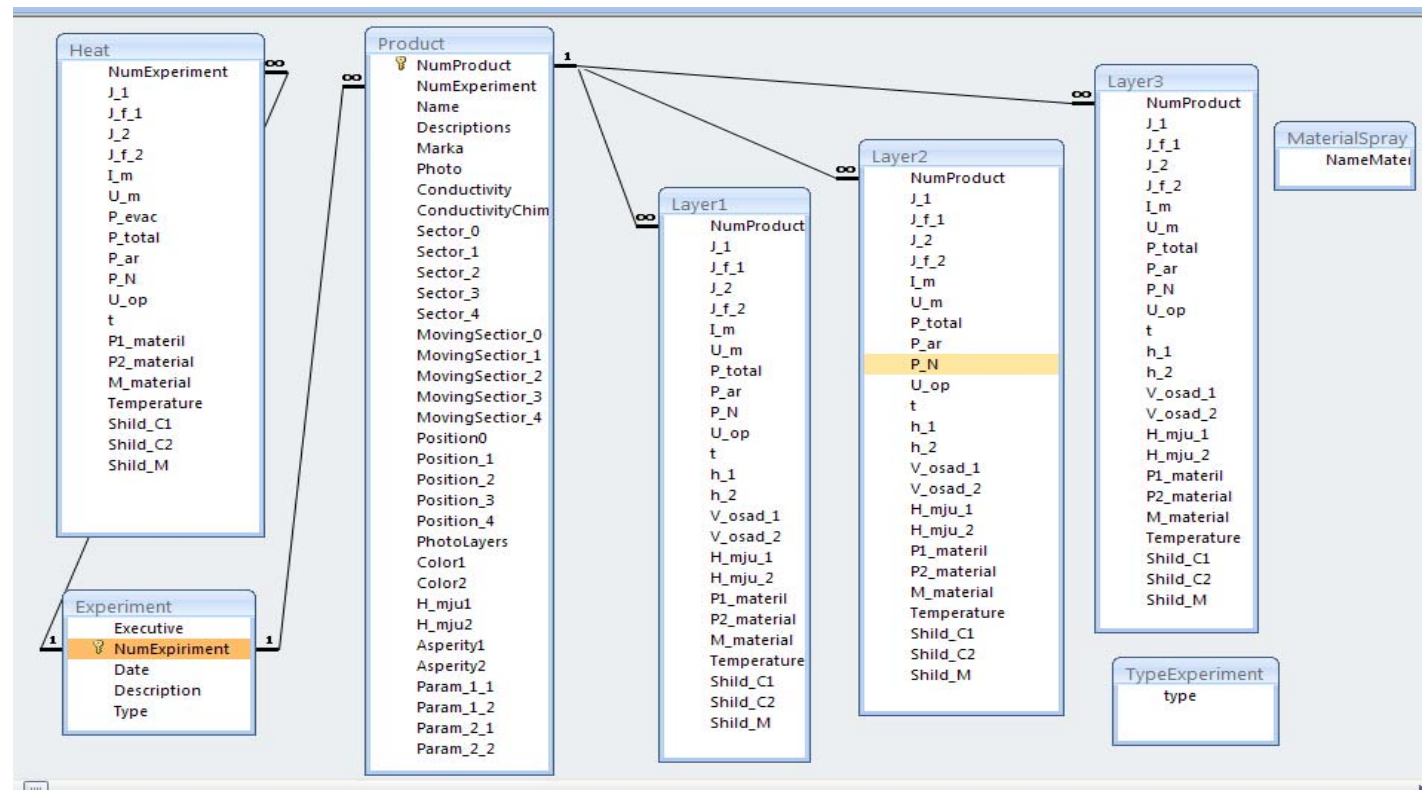

Figure 9. Database diagram

There has been developed and implemented the application interface. The user gets the main form, shown on Figure 10, allowing starting the operation with the database after running DBMS. There are the parameters for the experiment data registration in the upper part of the main form. There tab pages below; they provide the transit to the corresponding form.

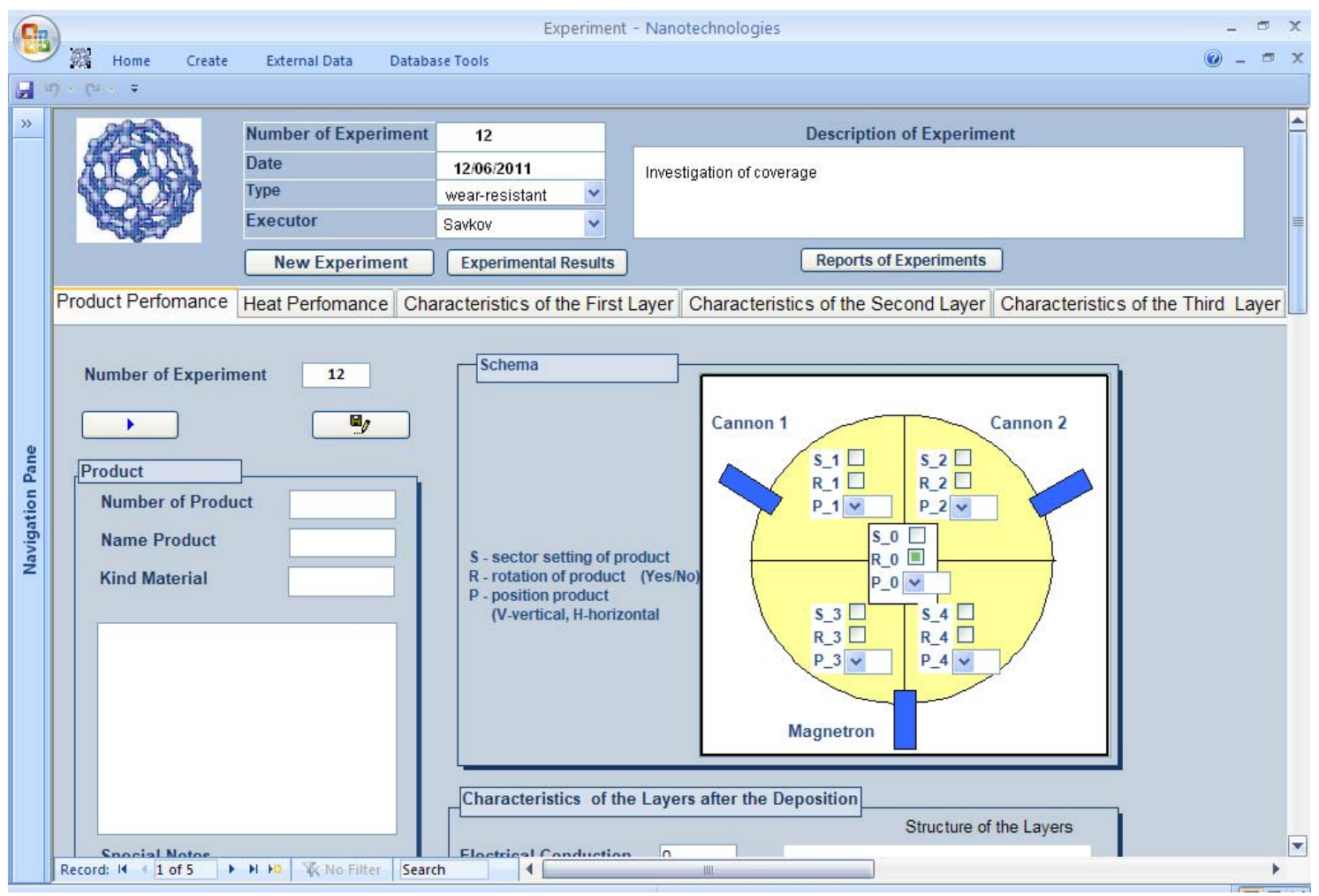

Figure 10. Application main form

There is the form for completing the data about the sample of experiment on the tab Product Performance (see Figure 10). The user employs Check Box to point in the diagram the sector location 
of the sample, its rotation round the axis and its position in the plant: vertical or horizontal. All the registered parameters are united into groups presenting the certain convenience for user, for instance, the parameters, registered after the experiment are united into group Characteristics of the Layers after Deposition. There is the form for introducing the data on heating mode on the tab Heat Performance (see Figure 11). The tabs Characteristics of the First Layers, Characteristics of the Second Layers Characteristics of the Third Layers contain the single-typed forms with parameters for accordingly the first, second and the third layers. These forms are shown on Figure 12.

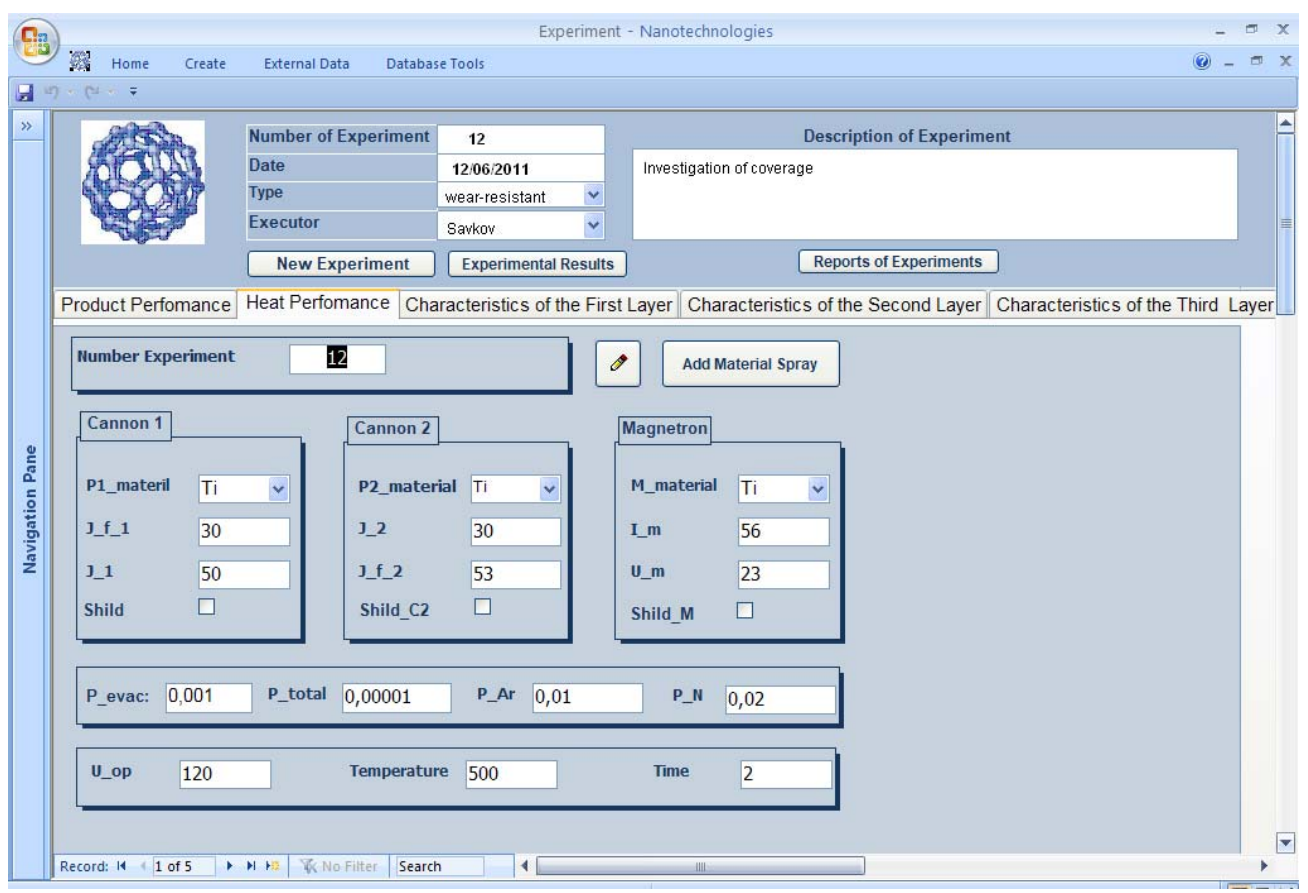

Figure 11. Form for introducing the data on the heating mode

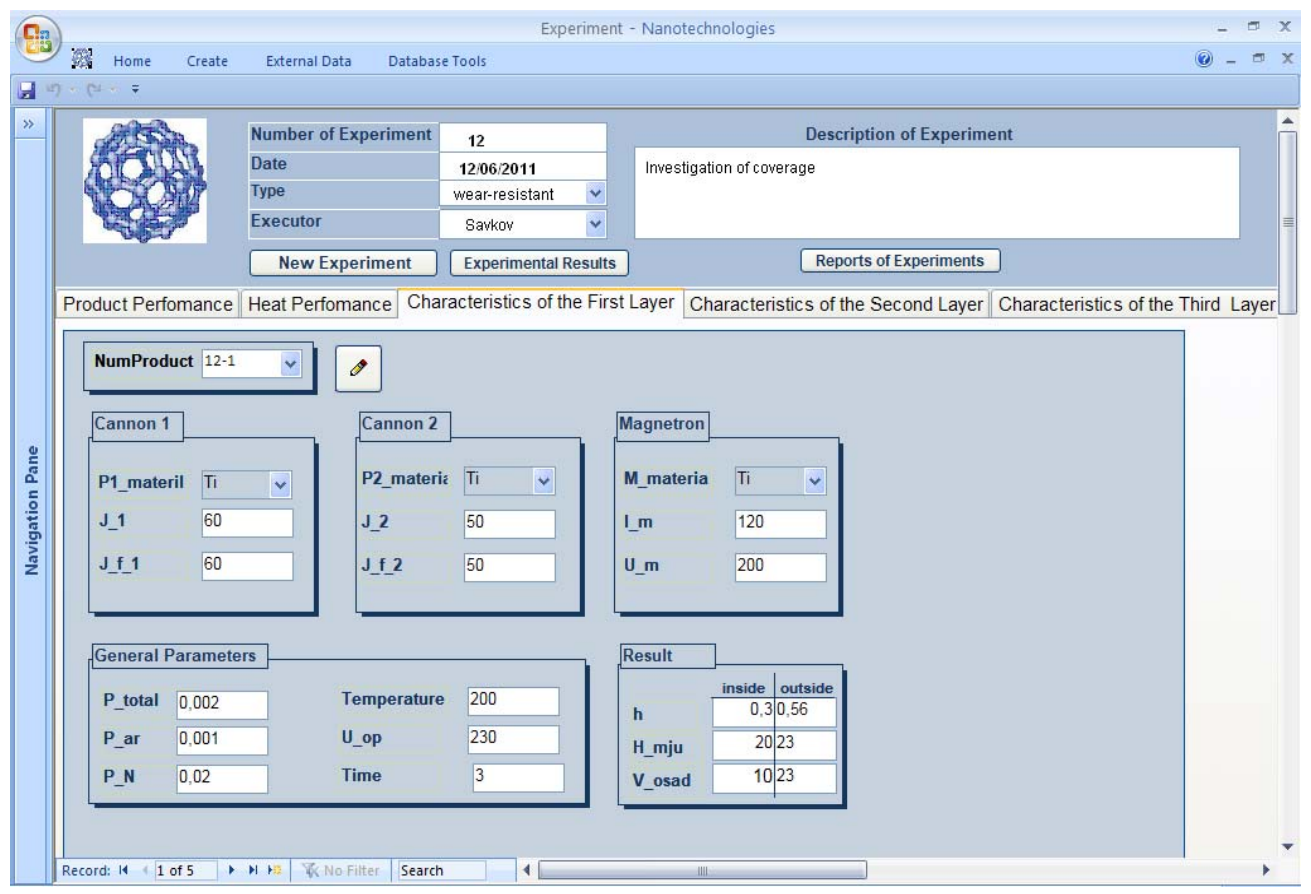

Figure 12. Form for introducing the data on the mode of applying the coating on the first layer 
There is a button Experimental Results on the main form, shown on Figure 13, which opens form "Results of Experiments". Employing this form, the user can view the experiments results for the certain time period, choose the experiments with specific material of deposition, and receive the data on experiments, resulted in certain level of hardness.

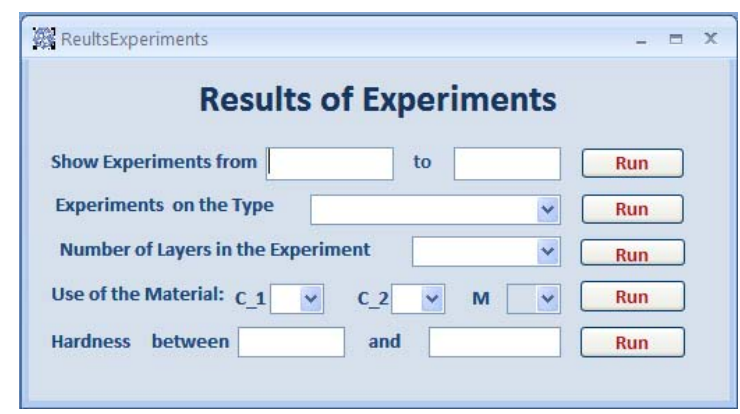

Figure 13. Protocol form

There is the button Report of Experiments on the main form for arranging the experiment protocol according to the settled form; it allows switching to the form "Report Experiment" (see Figure 14). It is possible to choose the number of experiment and then watch it in the preview mode or print it.

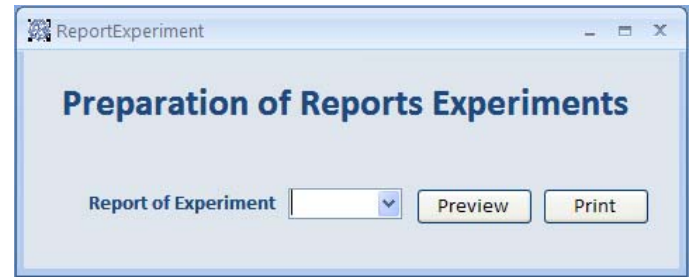

Figure 14. Report of Experiments Form

All the requirements towards the database declared in the stage of developing have been implemented; the developed database has been testing using real data.

\section{Analytical Processing the Results of Nanocoatings Properties Changes}

The different modes of the plant operation were set in the process of performing the experiments: pressure, voltage, current; two parameters were set as constant values, while the third parameter was changeable. The performed experiments resulted in the obtained dependencies of the coating settling velocity on the plant operation mode. The above presented graphs were obtained after the data processing in this analytical module, and the equations describe these dependencies. The demonstrated graphs are the results of the experiments investigating the procedure of applying the coating on the different sides of the gas-turbine engine blade (see Figure 15).
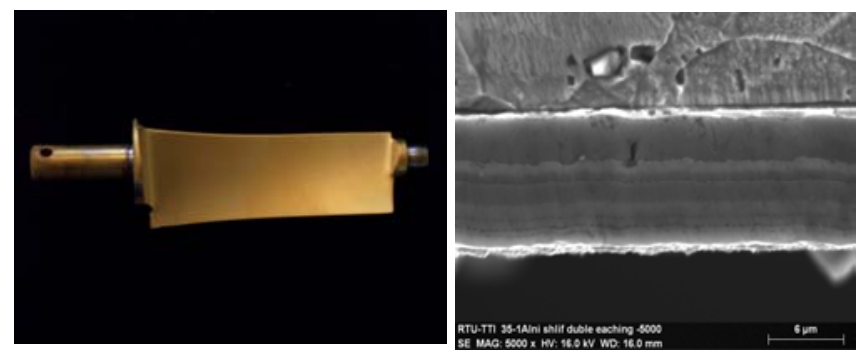

Figure 15. Stator blade of the gas-turbine engine after applying the coating and the view of the coating layer obtained with the electronic microscope employment 
The obtained dependencies allow the performer to specify the plant operation mode for receiving the certain properties of coating, for instance, the thickness of the coating. The determining parameter is the gas pressure in the plant, and then the coating settling velocity is determined according to the gas pressure. The coating settling velocity will determine the voltage and the current intensity in the plant employing the received dependencies are shown on Figure 16 and Figure 17.

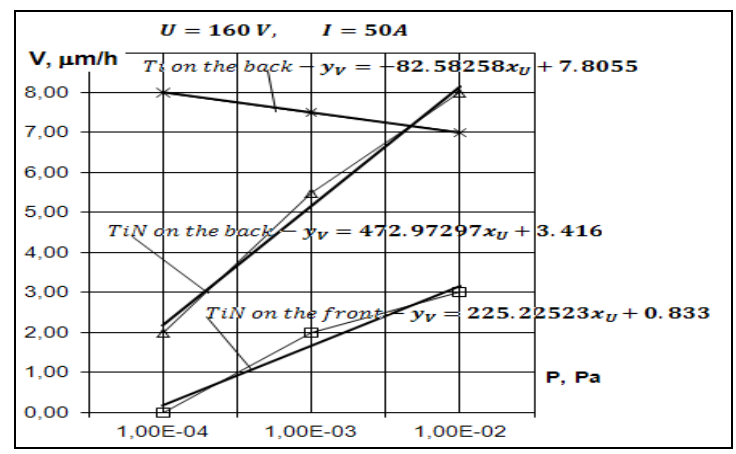

Figure 16. Dependence of coating settling velocity on the gas pressure in the plant

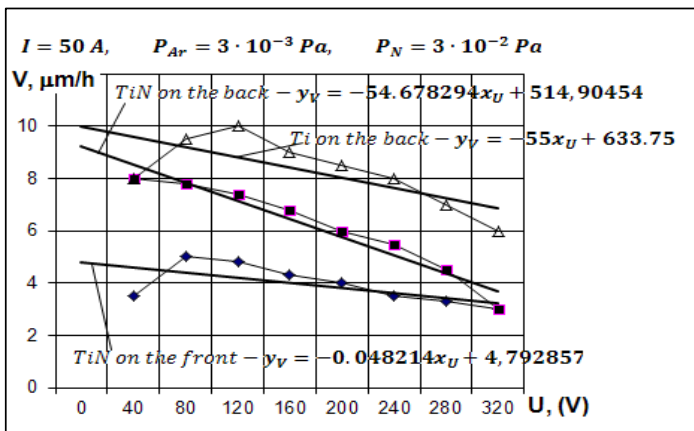

a)

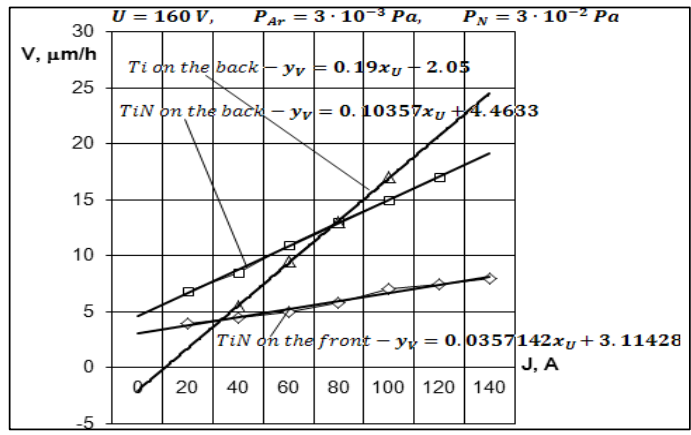

b)

Figure 17. Dependence of coating settling velocity on the voltage (a) and current intensity (b) in the plant

The analytical module allows identification of the plant mode parameters automatically and to a high precision, since it uses the equations, describing the obtained data. In the process of accumulating the experiments data in the different plant operation modes, the automatic correction of the received dependencies aimed at their updating and specification is done on the regular basis. Consequently, the analytical module allows efficient and prompt imposing the correcting procedures in the process of performing the experiment. Comparison of the forecast results and then this forecast verification has manifested a rather high rate of the results convergence.

\section{Conclusions}

The research under consideration covers the complex approach towards arranging the procedure of the experiments on applying the nanocoatings on various materials. This approach comprises the full provision of the experiments information support, including the database containing the entire information on the experiments results. The data kept in the database are "cleared" and then transferred to the data warehouse; consequently, the data are "downstocked" for the following analytical processing.

The researcher employing the system analytical module has possibility to analyse this information, discover the hidden dependencies, and make the results forecasts, and so on. This approach has provided the high quality level of performing the experiments on applying the nanocoatings on the surfaces of industrial products and machinery.

\section{Acknowledgements}

This article has been executed within the framework of the Joint research project of Latvian Council of Science "Development of Technology for Industrial Multicomponent Nanostructure Sheeting”. 


\section{References}

1. Uldrich, Jack (2006). Investing in Nanotechnology: Think Small. Win Big. Avon, MA USA: Adams Media Corporation.

2. Kopytov, E., Labendik, V., Yunusov, S., Urbach, A., Savkov, K. (2011). Informational Support of Carrying out Experiments on Producing Nano-coverings. In Proceedings of the VIII International Congress "Machines, Technologies, Materials 2011", Vol. 3, "Design \& Ergonomics, Industrial Informatics, Gear Transmission, Management”, September 19-21, 2011 (pp. 194-196), Bulgaria, Varna: Scientific-Technical Union of Mechanical Engineering.

3. Roco, Mihail C., Bainbridge, William S. (2001). Social Implications of Nanoscience and Nanotechnology. Arlington, Virginia: Kluwer Academic Publishers.

4. Lee, J. C, Wu, Y. F., Nee, T. E. (2011). Characterization of nanocrystallites of ingan/gan multiquantum wells by high-resolution X-ray diffraction. IEEE Transactions on Nanotechnology, 10(4), 827-831.

5. Urbaha, M., Savkov, R., Urbah, A. (2010). Development of Protective Coatings for Advanced Aero Engines. In Abstracts of the $2^{\text {nd }}$ International Symposium "Space \& Global Security of Humanity". 5-9 July, 2010 (p. 95). Riga, Latvia: TTI.

6. X-Ray Diffraction. (In Russian). Retrieved May 2, 2012 from http://www.xrd.ru/xrd6.html.

7. Kutev, V., Pozdnyakov, A., Krainyukov, A. (2011). A New Approach to Calculating X-ray Patterns of the Diffractometer for Nanostructured Coatings. In Proceedings of the VIII International Congress "Machines, Technologies, Materials 2011", Vol. 2: "Machines. Materials", September 19-21, 2011, (pp. 171-174), Bulgaria, Varna: Scientific-Technical Union of Mechanical Engineering, Bulgaria, Sofia.

8. Skiadas, C. H. and Skiadas, C. (2007). A modeling approach to life table data. In Recent Advances in Stochastic Modeling and Data Analysis (pp. 350-359). New Jersey, London, Singapore: World Scientific.

9. Skiadas, C. H., Dimotikalis, I. (2010). Chaotic Systems: Theory and Applications. New Jersey, London, Singapore: World Scientific.

10. Feldmann, Cl. G. (1998). The Practical Guide to Business Process Reengineering Using IDEF0. N.-Y.: Dorset House Publishing Co., Inc.

11. Groh, M. R., Stockmann, J. C. et al. (2007). Access 2007 Bible. Indianapolis: Wiley Publishing, Inc. 